\title{
Évaluation du comportement de quelques savons traditionnels en solution aqueuse : Détermination de la concentration micellaire critique et de la température de Krafft
}

TOGBE F. C. Alexis, YETE Pélagie, AZANDEGBE ENI Coffi, WOTTO D. Valentin.

Laboratoire de Chimie Physique (LCP/FAST/CHIMIE/UAC) 01BP526, Cotonou (Bénin).

Auteur correspondant, E-mail : abobosfr@yahoo.fr , Tel (00229) 96170488

Original submitted in on $13^{\text {th }}$ August 2014. Published online at www.m.elewa.org on $30^{\text {th }}$ November 2014. http://dx.doi.org/10.4314/iab.v83i1.3

\section{RÉSUMÉ}

Objectif: Cette présente étude a pour objectif de déterminer certaines grandeurs caractéristiques des savons traditionnels préparés au Benin en solution aqueuse.

Méthodologie et résultats : La concentration micellaire critique (CMC) et le point de Krafft des savons traditionnels sont déterminés par la méthode conductimétrique. Les résultats de cette étude conductimétrique ont révélé que tous les savons étudiés ont une $\mathrm{CMC}$ faible $(\mathrm{CMC}<3 \mathrm{~g} / \mathrm{L})$ et sont donc solubles et efficaces à température ambiante.

Conclusion et application: Ces savons traditionnels pourraient donc être classés dans la gamme des savons de ménage et également des savons de toilette.

Mots clés : Savons traditionnels, concentration critique micellaire, température de Krafft, Benin

\section{ABSTRACT}

Objective: This study aims to determine some characteristic quantities of traditional soaps prepared in Benin in aqueous solution.

Methods and Results: The critical micelle concentration (CMC) and the Krafft point of traditional soaps were determined by the conductivity method. The results of this study showed that conductivity of all the studied soaps had low $\mathrm{CMC}(\mathrm{CMC}<3 \mathrm{~g} / \mathrm{L})$ and therefore are soluble and effective at room temperature.

Conclusion and Application: These traditional soaps could be classified in the range of household soaps and toilet soaps.

Keywords: Traditional soaps, critical micelle concentration, Krafft temperature, Benin

\section{INTRODUCTION}

Parmi tous les tensioactifs connus jusqu'aujourd'hui, le plus ancien est le savon. Les premiers utilisateurs de savon auraient été les Sumériens en 2500 av. J.C. Ils fabriquaient les

pains de savon à partir d'huiles végétales ou animales, de cendre d'os ou de bois et d'extraits de plantes parfumées (Françoise B. \& Hélène C, 1993). L'apparition du savon, tel que connu 
aujourd'hui, aurait eu pour cadre la ville de Savone, en Italie, d'où il tire son nom. Pendant des siècles, le savon servait d'onguent, de cosmétique, de remède. II faut attendre le Moyen-âge pour que le savon soit utilisé pour laver le linge (Robert P. \& Jean-Pierre A, 1997]). Les tensioactifs sont des molécules d'intérêt dont le squelette amphiphile laisse apparaître des propriétés de surface spécifiques. Cette particularité structurale autorise leur usage pour la formulation de produits de consommation courante tels que les détergents et les cosmétiques, mais également dans de nombreuses industries comme le textile, le cuir, la métallurgie, ou encore les peintures (Geneviève $R$. \& Alain F, 1993). Le savon traditionnel appelé "KOTO" en langue nationale Fon et "AKOTO" en langue nationale Adja est un tensioactif ionique largement utilisé au Benin. Ces savons traditionnels sont préparés à base de cendres de végétaux et de matières grasses (Figure 1). Parfaitement connu, ce tensioactif est utilisé dans les produits domestiques en raison de ses propriétés moussantes et détergentes. L'étude des savons traditionnels en solution aqueuse permet de déterminer certaines grandeurs caractéristiques de ces savons telles que la concentration critique micellaire (Concentration à laquelle les molécules tensioactives forment de micelles en solution) et le point de Krafft (température à laquelle les molécules tensioactives forment de micelles en solution). Pour notre étude, la conductivité électrique des savons traditionnels dans l'eau a été déterminée. Pour cela, la méthodologie décrite dans les travaux antérieurs (Mehrotra et al, 1988 ; Mehrotra et al, 1994 ; Varma R P \& Abha K M, 1983 ; Varmar R P \& Goel H, 1994 ; Varma R P \& Kumar A, 2000 ; Varma A, 2000 ; Upadhyaya S K, 1997) est celle qui est utilisée. Signalons que ces auteurs n'ont déterminé les paramètres relatifs à la mise en solution de savons qu'à partir de savons purs synthétisés par double décomposition à partir de savons alcalins et de sels métalliques. Seuls certains auteurs ( Adebajo M O et al, 2001; Adeosun B F et al, 2001 ) font part d'études sur des savons issus de la saponification de triglycérides d'huile de palme.

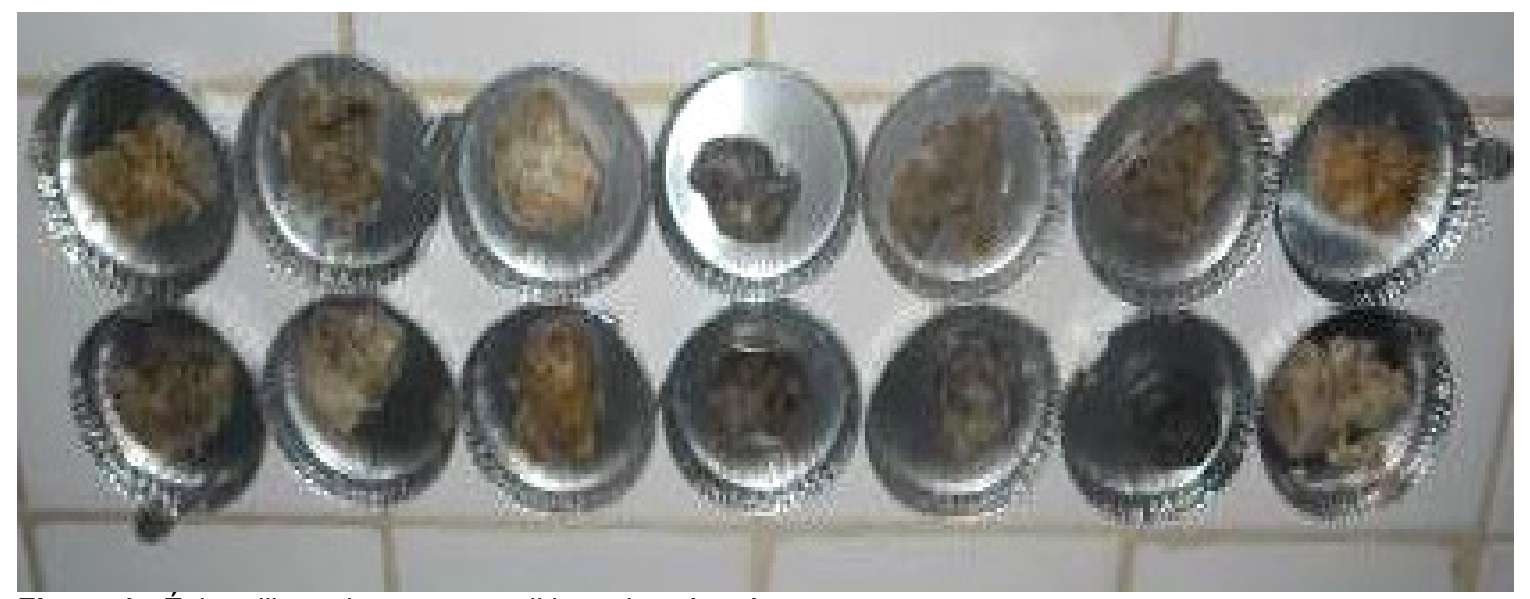

Figure 1 : Échantillons de savons traditionnels préparés

\section{MATÉRIEL ET MÉTHODES}

Préparation des échantillons de savons : $300 \mathrm{~g}$ de corps gras ont été mélangés avec $150 \mathrm{~g}$ de lessive de cendre dans un bol en acier inox. Le tout est porté à ébullition à une température de $70^{\circ}$ en mélangeant continuellement dans le même sens afin d'éviter que cela ne déborde jusqu'à ce que le savon se soit formé.
Puis on a laissé refroidi le mélange crémeux obtenu dans le bol jusqu'à solidification. Ainsi, par ce procédé 14 différents types d'échantillons de savons ont été fabriqués, avec comme corps gras l'huile de palme ou le beurre de karité mentionnés dans le tableau 1. 
Tableau 1 : Échantillons de savons préparés.

\begin{tabular}{lll}
\hline Lessive de cendres & Savons (Huile de palme) & Savons (Beurre de karité) \\
\hline$E_{1}$ & $E_{1 \mathrm{H}}$ & $E_{1 \mathrm{~B}}$ \\
$E_{2}$ & $E_{2 \mathrm{H}}$ & $E_{2 \mathrm{~B}}$ \\
$E_{3}$ & $E_{3 \mathrm{H}}$ & $E_{3 \mathrm{~B}}$ \\
$E_{4}$ & $E_{4 \mathrm{H}}$ & $E_{4 \mathrm{~B}}$ \\
$E_{5}$ & $E_{5 \mathrm{H}}$ & $E_{5 \mathrm{~B}}$ \\
$E_{6}$ & $E_{6 \mathrm{H}}$ & $E_{6 \mathrm{~B}}$ \\
$E_{7}$ & $E_{7 \mathrm{H}}$ & $E_{7 \mathrm{~B}}$ \\
\hline
\end{tabular}

$E_{1 H} a ̀ E_{7 B}$ : code des échantillons de savons analysés

Ensuite, ces différents échantillons ont été sélectionnés suivant le $\mathrm{pH}$ et le taux d'humidité (teneur en eau et matières volatile). Les échantillons ayant un $\mathrm{pH}$ compris entre 8,5 et 10 et un taux d'humidité inférieur à $34 \%$ ont fait l'objet d'autres analyses (volume de mousse, teneur en alcali libre caustique et en alcali libre total).

Détermination du $\mathrm{pH}$ : Le $\mathrm{pH}$ des échantillons de savons a été déterminé avec le papier pH (marque MERCK).

Détermination de la teneur en eau et des matières volatiles : La méthode par étuvage a été utilisée selon la norme NF T 60-305 de juin 1993.

Détermination du volume de mousse : $\mathrm{A} 0,01 \mathrm{~g}$ près, $0,25 \mathrm{~g}$ d'échantillon de savon a été pesé dans une éprouvette de $100 \mathrm{ml}$ dans laquelle on a ajouté $25 \mathrm{ml}$ d'eau. L'ensemble a été soumis pendant 1 à 2 minutes à une agitation à l'aide d'un agitateur vibra mixer, puis le volume de mousse a été déterminé. N.B. : Le poids du savon $(0,25 \mathrm{~g})$ et le volume d'eau $(25 \mathrm{ml})$ utilisés sont des quantités qui ont permis, dans nos conditions expérimentales, d'obtenir les meilleurs résultats.

Détermination de la teneur en alcali libre total : La teneur en alcali libre total des savons est déterminée suivant la norme NF T60-308. Elle est mesurée par la dissolution du savon dans une solution éthanolique et neutralisation de l'alcali libre par une solution d'acide sulfurique dont l'excès connu est titrée en retour par une solution éthanolique d'hydroxyde de potassium.

Détermination de la CMC par conductivité : Dans des béchers, une solution de chaque échantillon de

\section{RÉSULTATS ET DISCUSSION}

La micellisation des savons étudiés précédemment ont été étudié par conductivité. La température de l'expérience est choisie en fonction de la température savon de concentration $5 \mathrm{~g} \cdot \mathrm{L}^{-1}$ a été préparée un jour à l'avance afin qu'elle se stabilise pour éviter la formation de mousse. Cette solution a été utilisée afin de titrer $100 \mathrm{ml}$ d'eau déminéralisée à température ambiante.

La conductivité de chaque solution de savon a été mesurée à chaque fois $5 \mathrm{ml}$ d'eau dés ionisée ont été ajoutés. La concentration micellaire critique est déterminée après la lecture de la courbe de la conductivité en fonction de la concentration de notre solution. La conductivité a été mesurée avec un conductimètre doté d'une sonde WTW 340i.

Détermination de la température de Krafft: La température de Krafft a été déterminée dans la solution de chaque échantillon de savons de concentration égale à $5 \mathrm{~g} . \mathrm{L}^{-1}$. Comme précédemment, la conductivité de la solution qui est dans un bêché et mise sur un agitateur chauffant a été mesurée. Cette fois-ci, les mesures ont commencé à partir d'une température d'environ $10^{\circ} \mathrm{C}$ puis on fait chauffer la solution jusqu'à $90^{\circ} \mathrm{C}$ environ, le tout en agitant la solution afin que tout le tensioactif soit bien solubilisé. La solution étant placée au réfrigérateur pendant environ 15 minutes avant la manipulation pour que le tensioactif tombe au fond du récipient afin de faciliter sa dissolution et d'éviter qu'il ne s'agglomère sur la sonde. Les mesures de conductivité ont été prises tous les $5^{\circ} \mathrm{C}\left( \pm 0,1^{\circ} \mathrm{C}\right)$.

Analyses statistiques des résultats: Les analyses statistiques (analyse de la variance, intervalle de confiance de la moyenne) ont été réalisées par le logiciel Microsoft Word " 2007" Les valeurs obtenues sont des moyennes de 3 répétitions

de Krafft de chaque savon qui a été déterminée au préalable afin de s'assurer que le tensioactif est soluble. Avec la technique conductimétrique, la 
Togbe et al.. J. Appl. Biosci. 2014 Évaluation du comportement de quelques savons traditionnels en solution aqueuse : Détermination de la concentration micellaire critique et de la température de Krafft

température de Krafft correspond au changement abrupt de la pente sur le graphique de conductivité en fonction de la température (Zana R et al, 2004;

Tableau 2 : Point de Krafft des savons préparés

\begin{tabular}{lllllllll}
\hline Savons & $\mathrm{E}_{1 \mathrm{H}}$ & $\mathrm{E}_{1 \mathrm{~B}}$ & $\mathrm{E}_{2 \mathrm{H}}$ & $\mathrm{E}_{2 \mathrm{~B}}$ & $\mathrm{E}_{4 \mathrm{H}}$ & $\mathrm{E}_{4 \mathrm{~B}}$ & $\mathrm{E}_{5 \mathrm{H}}$ & $\mathrm{E}_{5 \mathrm{~B}}$ \\
\hline $\mathrm{T}_{\mathrm{K}}\left({ }^{\circ} \mathrm{C}\right)$ & 26 & 25 & 25 & 26 & 26 & 26 & 24 & 26 \\
\hline
\end{tabular}

La polarité du solvant guide la conductivité : un solvant ionisant, polarisant et solvatant tel que l'eau exerce des forces diélectriques favorables à l'ionisation des molécules, à la solvatation et à la mobilité des ions. Ainsi, le couple savon/eau permet d'obtenir des valeurs de la concentration micellaire critique $(\mathrm{CMC})$ en fonction de la température ambiante $\left(25^{\circ} \mathrm{C}\right)$. Quel que
Fergoug $T$ et al, 2004). Le tableau 2 rassemble les points de Krafft $\left(T_{K}\right)$ déterminés dans le cadre de ce travail au niveau de chaque savon. soit le solvant utilisé, les courbes donnant la conductivité en fonction de la concentration du savon sont caractérisées par l'intersection de deux droites intersection donne la CMC du savon dans l'eau, et pour chaque couple savon/eau, la CMC est déterminée et donnée dans le tableau 3. (figure 2). La concentration correspondante à cette

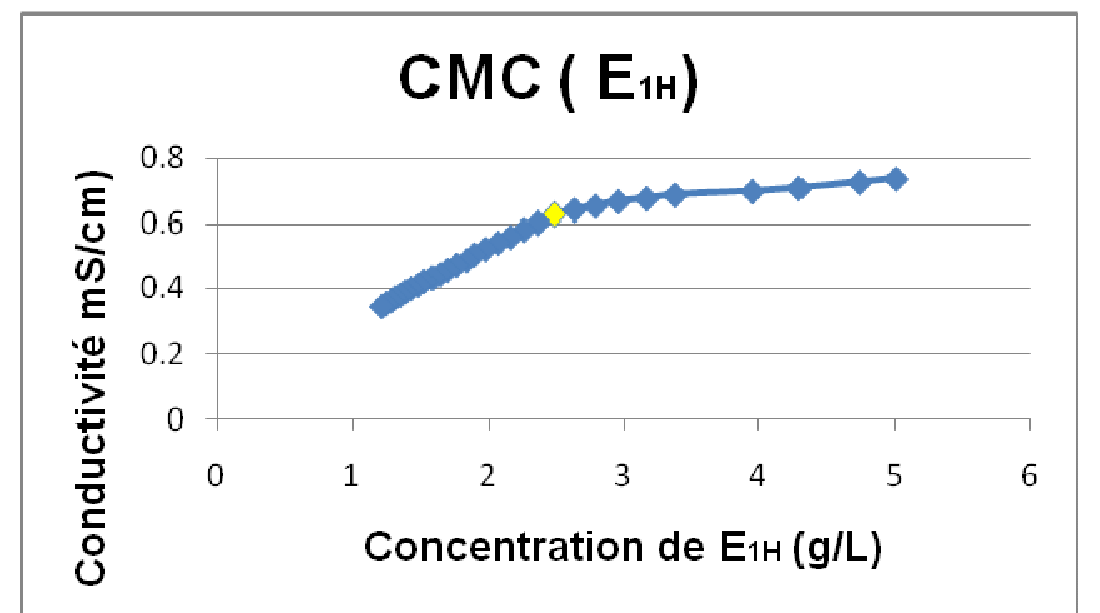

Figure 2: Variation de la conductivité du savon $\mathrm{E}_{1 \mathrm{H}}$ en fonction de sa concentration à $\mathrm{T}=26^{\circ} \mathrm{C}$

Tableau 3 : CMC des savons traditionnels étudiés.

\begin{tabular}{lll}
\hline Savons & Température $\left({ }^{\circ} \mathrm{C}\right)$ & CMC $(\mathrm{g} / \mathrm{L})$ \\
\hline$E_{1 H}$ & 26 & 2,49 \\
$E_{1 B}$ & 25 & 2,53 \\
$E_{2 H}$ & 25 & 2,56 \\
$E_{2 B}$ & 26 & 2,36 \\
$E_{4 H}$ & 26 & 1,79 \\
$E_{4 B}$ & 26 & 2,70 \\
$E_{5 H}$ & 24 & 2,48 \\
$E_{5 B}$ & 26 & 2,25 \\
\hline
\end{tabular}

L'analyse du tableau 2 révèle que tous les savons ont une température de Krafft voisine de la température 


\section{Togbe et al.. J. Appl. Biosci. 2014 Évaluation du comportement de quelques savons traditionnels en}

solution aqueuse : Détermination de la concentration micellaire critique et de la température de Krafft

valeur, chaque savon est suffisamment soluble pour s'organiser et produire des micelles. C'est à partir de la température de Krafft que la micellisation est initiée. L'allure de la courbe de la figure 2 obtenue correspond aux attentes décrites dans la littérature. On observe nettement qu'au début, la conductivité électrique est une fonction linéaire de la concentration. Le changement se fait à une certaine concentration qui correspond à la $\mathrm{CMC}$ qui est ici égale à $2,49 \mathrm{~g} / \mathrm{L}$ pour le savon $E_{1 H}$. Les $C M C$ de tous les savons étudiés sont présentées dans le tableau 3 . La conductivité augmente en fonction de la concentration en tensioactif car la conductivité d'un tensioactif ionique avant la CMC est celle d'un électrolyte fort à une variation linéaire. Après la $C M C$, un brusque changement de plusieurs propriétés physiques dont la conductivité a été observé. En effet, la formation de micelles affecte la conductivité des tensioactifs car les contre ions deviennent une partie de la micelle, ce qui réduit le nombre de contre ion libres participant au transport du courant (Anouti M et al, 2009). A faible concentrations, l'augmentation de la conductivité en fonction de la concentration est due à l'ionisation des molécules de savons en cations $\left(\mathrm{K}^{+}, \mathrm{Na}^{+}, \mathrm{Mg}^{2+}, \mathrm{Ca}^{2+}\right)$ et en anions carboxylates lipophiles (RCOO-). La CMC déterminée par ces études correspond à la formation de micelles ioniques due à l'agrégation d'ions (Varade D et al, 2005 ; Anouti $\mathrm{M}$ et al, 2009 ). L'analyse du tableau 3 révèle que tous les savons ont une CMC relativement faible et donc qu'ils sont efficaces à faible concentration. En effet, l'agrégation des molécules débute lorsque l'énergie libérée lors de l'association des chaines hydrocarbonées de savon est suffisante pour compenser la répulsion électrique existant entre les groupements de tête ionique ainsi que la diminution

\section{CONCLUSION}

Au cours de ce travail, quelques caractéristiques des savons traditionnels analysés en solution aqueuse ont été étudiées. La concentration micellaire critique et le point de Krafft de ces savons ont été déterminés par conductimétrie. Les résultats de cette étude conductimétrique des solutions de savon ont révélé que

\section{REMERCIEMENTS}

Ce travail de recherche a été réalisé grâce au financement du programme de soutien du Ministère de l'Enseignement Supérieur et de la Recherche d'entropie qui accompagne cette agrégation. Les valeurs de concentration auxquelles l'agrégation commence augmentent avec la température car l'énergie cinétique des monomères augmente aussi avec cette grandeur (Varade D et al, 2005). Adebajo M 0 et al, 2001) a montré que la présence d'impureté diminue la mobilité des porteurs de charges. Dans notre cas, compte tenu de la source de nos alcalis (lessives de cendres végétales), les principales impuretés seraient donc dues à la présence du glycérol et de la base libre non consommée lors de la réaction de saponification. Le glycérol n'est pas une substance conductrice (glycérol pur : $0,1-1,0^{*} 10^{-3} \mathrm{mS} / \mathrm{cm}$ entre 25 et $90^{\circ} \mathrm{C}$ ), ce n'est donc pas lui qui influe sur la conductivité de la solution de savon brut. En revanche la base issue des lessives de cendres végétales sont composées de différents contre ions et sont donc très conductrices. En effet, (Zana R et al, 2004) ont montré que ces contre ions sont fortement liés à la micelle et provoquent une diminution de la répulsion électrostatique des têtes polaires, ce qui est responsable de la faible concentration de la CMC observée. II est à noter qu'un notre paramètre qu'est la longueur de l'espaceur pourrait également être aussi à l'origine de cette baisse de CMC. (Adeosun B F et al, 2001) ont étudié les couples savon/eau et savon/éthanol et s'agissant du couple savon/eau il ont observé le même phénomène. L'eau étant un solvant polaire, tous nos les savons ont alors tendance a y être dissociés. Les agrégats de savons formés sont de taille plus petite ce qui engendre une conductivité de la solution plus forte. II est clair que la conductivité élevée des solutions de savon ne provient pas de la conductivité inhérente à l'eau, mais est bien due à la dissociation du savon dans l'eau (Salager J-L, 1993).

tous les savons étudiés ont une $\mathrm{CMC}$ faible $(\mathrm{CMC}<3$ $\mathrm{g} / \mathrm{L}$ ) et sont solubles et efficaces à température ambiante. Un bon nombre de facteurs tels que la longueur de chaîne du tensioactif ou la température ont une influence sur la CMC et l'efficacité des savons.

Scientifique aux universités nationales du Bénin. A cet effet j'exprime mes remerciements et ma profonde gratitude au gouvernement du BENIN. 


\section{RÉFÉRENCES}

Adebajo M O, Akanni MS, 2001.The Electrical Conductance and Viscosity of Nigerian Traditional Soap in Alcoholic. Media. Colloids Surf. Physicochem. Eng. 194(2): 97-110.

Adeosun B F, Akande T, Oyewusi P A, Akinyele S A, 2001. Preliminary Studies on the Electrical Conductance and the Viscosity of some Nigerian Traditional Soap in Aqueous Solution. Pakist. J. Sci. Ind. Res. 44(6): 315320.

Anouti M, Jones J, Boisset A, Jacquemin J, CaillonCaravanier M, Lemordant D, 2009. Aggregation behavior in water of new imidazolium and pyrrolidinium alkycarboxylates protic ionic liquids. Journal of Colloid and Interface Science. 340: 104-111.

Fergoug T, Bendedouch D, Aicart E, 2004. Characterization of the 1heptodecafluorodecylpyridinium iodide in solution: Partial phase diagram and micellar properties from conductivity and surface tension. Colloids and Surfaces A: Physicochemical and Engineering Aspects. 237: 95-103

Françoise B, Hélène $C$, 1993. « Monuments des eaux » et «Toilette », dans Quand les Gaulois étaient Romains, Paris, Découvertes Gallimard 63, p. 47 à 49 et p. 105 à 108.

Geneviève R, Alain F. 1993. Costumes et textiles en Gaule romaine, Paris, Errance, 175 p. ill.

Mehrotra K N et Upadhyaya S K, 1988. Study on Solubility, Viscosity and Conductivity of Calcium Soaps. Tenside Surfact. Deterg., 25(5):302-307

Mehrotra K N, Rani L et Kumar A, 1994. PhysicoChemical Propeties of Lithium Soaps. Rev. Roum. Chim. 39(5): 517-525
Robert P, Jean-Pierre S, 1997. Chimie industrielle. 2eme édition. $P$ : 679-685.

Salager J-L, 1993. Surfactifs en solution aqueuse. Laboratorio FIRP,Escuela de Ingeniería Química, Universidad de Los Andesn Mérida5101 VENEZUELA

Upadhyaya S K, 1997. Conductance Behaviour and Thermodynamics of Dissociation and Micellization of Calcium Palmitate and Stearate. Indian J.Chem .36A(12):1054-1057

Varade D, Joshi T, Aswal V K, Goyal P S, Hassan P A, Bahadur $P, 2005$. Effect of salt on the micelles of cetyl pyridinium chloride. Colloids and Surfaces A: Physicochemical and Engineering Aspects. 259: 95-101.

Varma A, 2000. Conductivity of Strontium Soap in Water. Asian J. Chem. 12(2):407-411

Varma R P et Abha K M, 1983. Investigation into the Conductivity of Magnesium Caprate in Aqueous Alcohols. Tenside Deterg. 20(4): 196-198

Varma R P et Bahadur P, 1975. Studies on Conductivity of Critical Micelle Concentration and Study of Physical Properties of Aqueous Solutions of Calcium Soap. Cellul. Chem. Technol. 9(4):393-401

Varma R P, Kumar A, 2000. Characterization of sodium Soap and their Refractive Index Studies in Methanol. Asian J. Chem. 12(3): 659-662

Varmar R P et Goel H, 1994. Conductance Behaviour of Lithium Soap in Water and Methanol. Coll. Surf. A Phys. Eng. Asp.85:69-73

Zana R, Benrraou M, Bales B L, 2004. J. Colloid Interface Sci. 80: 224. 